\title{
From Type-II Triply Degenerate Nodal Points and Three-Band Nodal Rings to Type-II Dirac Points in Centrosymmetric Zirconium Oxide
}

\author{
Ting-Ting Zhang, ${ }^{1,2}$ Zhi-Ming Yu, ${ }^{3, *}$ Wei Guo, ${ }^{4}$ Dongxia Shi, ${ }^{1,2,5}$ Guangyu Zhang,,${ }^{1,2,6,5, \dagger}$ and Yugui Yao ${ }^{4, \ddagger}$ \\ ${ }^{1}$ Institute of Physics, Chinese Academy of Sciences, Beijing 100190, China \\ ${ }^{2}$ School of Physical Sciences, University of Chinese Academy of Sciences, Beijing 100190, China \\ ${ }^{3}$ Research Laboratory for Quantum Materials, Singapore University of Technology and Design, Singapore 487372, Singapore \\ ${ }^{4}$ School of Physics, Beijing Institute of Technology, Beijing 100081, China \\ ${ }^{5}$ Beijing Key Laboratory for Nanomaterials and Nanodevices, Beijing 100190, China \\ ${ }^{6}$ Collaborative Innovation Center of Quantum Matter, Beijing 100190, China
}

\begin{abstract}
Using first-principles calculations, we report that $\mathrm{ZrO}$ is a topological material with the coexistence of three pairs of type-II triply degenerate nodal points (TNPs) and three nodal rings (NRs), when spin-orbit coupling (SOC) is ignored. Noticeably, the TNPs reside around Fermi energy with large linear energy range along tilt direction $(>1 \mathrm{eV})$ and the NRs are formed by three strongly entangled bands. Under symmetry-preserving strain, each NR would evolve into four droplet-shaped NRs before fading away, producing distinct evolution compared with that in usual two-band NR. When SOC is included, TNPs would transform into type-II Dirac points while all the NRs are gaped. Remarkably, the type-II Dirac points inherit the advantages of TNPs: residing around Fermi energy and exhibiting large linear energy range. Both features facilitate the observation of interesting phenomena induced by type-II dispersion. The symmetry protections and low-energy Hamiltonian for the nontrivial band crossings are discussed.
\end{abstract}

\section{INTRODUCTION}

The exploration of topological materials has now extended from topological insulators ${ }^{1,2}$ to topological semimetals, such as Weyl ${ }^{3-5}$ and Dirac semimetals ${ }^{6-9}$. In Weyl (Dirac) semimetals, the low energy electrons residing around Weyl (Dirac) point obey relativistic equation, providing the possibility to simulate intriguing highenergy physics in solids ${ }^{10,11}$. Currently, the discovery of type-II Weyl semimetals ${ }^{12,13}$ unveils that in solids, the kinds of quasiparticles would be more abundant than that in high-energy physics, due to the reduced symmetry constraint. Soon after, type-II Dirac point and multi-fold (beyond doubly and fourthly) degenerate nodal point are successively proposed ${ }^{14-23}$. All the new fermions exhibit physical phenomena distinguished from each other and conventional Weyl and Dirac fermions ${ }^{21,22,24,25}$. Many materials are predicted to be the candidates for hosting the new fermions and some of them are confirmed by experiment, such as, $\mathrm{MoTe}_{2}{ }^{13,26}\left(\mathrm{PtTe}_{2}{ }^{27-30}\right)$ families are confirmed as type-II Weyl (Dirac) semimetal and $\mathrm{MoP}^{31}$ is confirmed as type-I TNP semimetal. However, searching for ideal semimetals ${ }^{32}$ with nodal point locating at Fermi energy and exhibiting large linear energy range is still desirable.

Besides the topological semimetals with zero dimensional band crossing, NR semimetals, featuring onedimensional band crossing $^{33-43}$, also have attracted tremendous attention. Intuitively, similar to Weyl semimetal, a two band model is enough to capture the low-energy physics of (doubly degenerate) NR. The twoband model works well for most reported NR materials. However, a recent work shows that the $\mathrm{NR}$ in $\mathrm{TiB}_{2}{ }^{44}$ is distinct from conventional two-band $\mathrm{NR}$ in that its formation requires a four-band model, as the two bands forming NR are strongly entangled with other two bands.
Hence, the $\mathrm{NR}$ in $\mathrm{TiB}_{2}$ are termed as four-band $\mathrm{NR}^{44}$. Furthermore, the evolution of two-band NR and fourband NR under strain can be very different ${ }^{44,45}$. Consequently, one can expect that other multi-band NR may lead to many distinct phenomena compared with conventional two-band NR and may be considered as a new kind of intriguing topological semimetal to be discovered.

In this work, using first-principles calculations and symmetry analysis, we show that $\mathrm{ZrO}$ is a topological metal with the coexistence of three pairs of type-II TNPs and three NRs, when SOC is ignored. The type-II TNPs locate at three high-symmetry lines and are protected by the $C_{4 v}$ point group symmetry. TNPs have been predicted in many materials ${ }^{18-20,22,44}$. However, typeII TNP semimetal is rare ${ }^{14}$. Compared with previously studied TNP semimetals, the TNPs identified here has several advantages, such as it has type-II dispersion, its nodal energy is close to Fermi energy and its linear energy range is large. Regarding to the three NRs, we find that they lie in three mirror planes, respectively and hence are doubly protected by mirror symmetry and the combination of time reversal $(\mathcal{T})$ and inversion $(\mathcal{P})$ symmetry. Interestingly, the formation of the NRs requires three bands rather than two bands and hence we term the NRs here as three-band nodal ring (TNR). Moreover, it is found the three bands forming TNRs are exactly the bands forming TNPs. In the following, we will see that the appearance of TNRs is closely related to the TNPs and the coexistence of TNR and TNP can be found in many materials ${ }^{22,44}$. Particularly, under symmetrypreserving strain, e.g. hydrostatic strain, each TNR here would evolve into four droplet-shaped nodal rings before vanishing, distinguished from previously studied nodal rings. When SOC is included, all the TNRs are gapped while each type-II TNP becomes a type-II Dirac point due to the presence of $\mathcal{P}$ and $\mathcal{T}$ symmetry. Remarkably, 


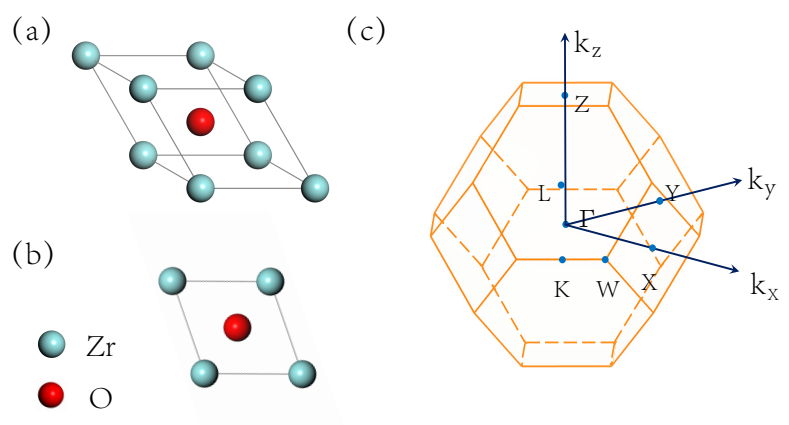

FIG. 1. (a) Side view and (b) top view of the crystal structure of FCC-type $\mathrm{ZrO}$. (c) The bulk Brillouin zone of $\mathrm{ZrO}$.

all the type-II Dirac points almost locate at Fermi energy with large linear energy range. Thus, $\mathrm{ZrO}$ would be an ideal platform for studying the interesting physics induced by type-II dispersion.

\section{METHOD AND CRYSTAL STRUCTURE}

We have employed the Vienna ab initio simulation package (VASP) ${ }^{46}$ for most of the first-principle calculations. The potentials are treated with the projector augmented wave (PAW) method ${ }^{47}$. Exchangecorrelation potential is treated within the generalized gradient approximation (GGA) of the Perdew-BurkeErnzerhof type ${ }^{48}$. The cutoff energy is chosen as $520 \mathrm{eV}$ and a $21 \times 21 \times 21 \Gamma$-centered $k$-mesh for self-consistent calculations. The energy convergence criterion is set to be $10^{-6} \mathrm{eV}$. The crystal structure is fully relaxed until the maximum force on each atom was less than $0.01 \mathrm{eV} / \AA$.

The elements $\mathrm{Zr}$ and $\mathrm{O}$ can form different kinds of zirconium oxide ${ }^{49,50}$. $\mathrm{ZrO}$ can be synthesized at proper oxygen atmosphere and shows FCC-type cubic crystal structure with space group $F m \overline{3} m$ (No. 225, $O_{h}^{5}$ ) as shown in Fig. 1. $\mathrm{Zr}$ and $\mathrm{O}$ atoms occupy the $(0,0,0)$ and $(0.5,0.5,0.5)$ Wyckoff positions, respectively. The experimental lattice constants are $a=b=c=3.254 \AA$. The optimized lattice constants are $a=b=c=3.271 \AA$, which are very closed to experimental values, being overestimated by about $0.5 \%$ and all the results discussed in the following are from the calculations with optimized structures. Figure 1(c) shows the bulk Brillouin zone (BZ) of $\mathrm{ZrO}$ crystal.

\section{TRIPLY DEGENERATE NODAL POINT}

The electronic band structure of $\mathrm{ZrO}$ without $\mathrm{SOC}$ is ploted in Fig.2(a), showing a metallic phase with highly dispersive bands. The band crossing point $T$ on $\Gamma-X$ path is especially striking, as it is close to Fermi energy
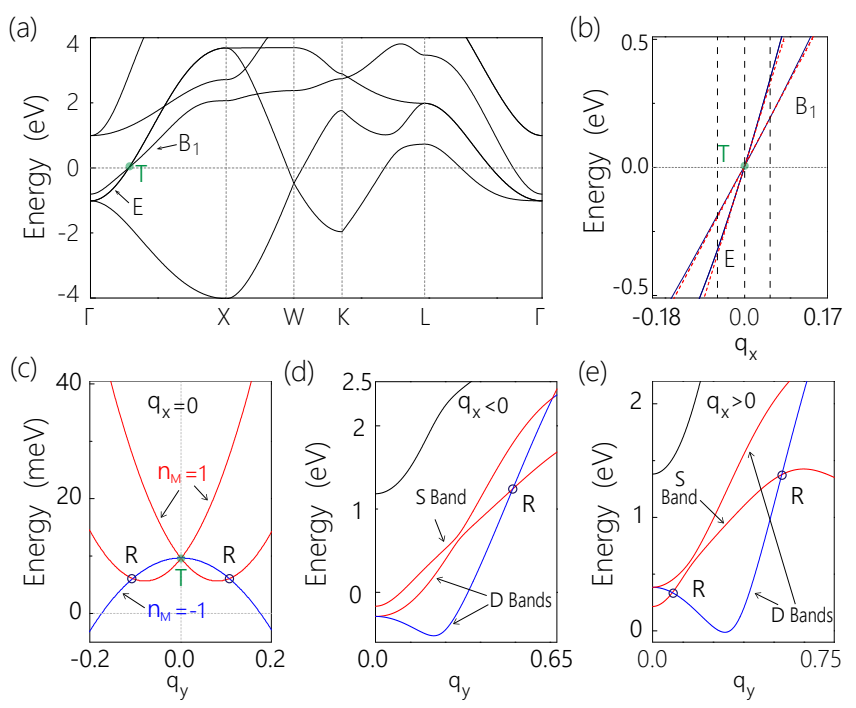

FIG. 2. (a) Calculated electronic band structure of $\mathrm{ZrO}$ without SOC. The crossing point on $\Gamma-X$ line is triply degenerate, labeled as $T$. The crossing bands have different IRs of $C_{4 v}$ point group: $E$ and $B_{1}$. (b) Dispersion around the TNP (T point) by first-principles calculations (solid lines) and $k \cdot p$ model fitting (red dashed lines). (c), (d) and (e) are the band dispersion along $q_{y}$ direction in the $q_{z}=0$ plane for different $q_{x}$ marked by the three black dashed lines in (b). The two mirror eigenvalues of $\mathcal{M}_{x y}\left(n_{M}=1\right.$ and $\left.n_{M}=-1\right)$ of the relevant three bands are denoted by red and blue color, respectively. The crossing points belonging to TNR are labeled as $R$. Here, the unit of $q_{x(y)}$ is $\pi / a(\pi / b)$.

and features large linear energy range $(>1 \mathrm{eV})$. On $\Gamma-X$ path, the litter point group is $C_{4 v}$. And the two bands formed point $T$ belong to two distinct irreducible representations (IRs) of $C_{4 v}: B_{1}$ (one-dimensional IR) and $E$ (two-dimensional IR), respectively (see Tab.I). Therefore, the band crossing is triply degenerate and is protected by $C_{4 v}$ symmetry. Moreover, the slope of the two crossing bands share same sign. Thus, point $T$ is a type-II TNP. Due to the three $C_{4 v}$ symmetries along $k_{x}, k_{y}$ and $k_{z}$ axes, $\mathrm{ZrO}$ exhibits three pairs of type-II TNP residing on three axes. In the following, we use the point $T$ to discuss the properties of TNP. Besides, the band crossing at $\mathrm{W}$ point is also obvious, which is about $0.47 \mathrm{eV}$ below Fermi energy. This band crossing is doubly degenerate and is essential, as its IR is $E$ of the litter group of W point $D_{2 d}$.

To characterize the low-energy physics of type-II TNPs, we establish a $k \cdot p$ model in the vicinity of point $T$ using bands with $B_{1}$ and $E$ IRs as basis. The Hamiltonian around $T$ up to linear order in $\boldsymbol{q}$ (measured from $T$ point) reads (see Supporting Information for details)

$$
\mathcal{H}_{T}=C_{1} q_{x}+\left(\begin{array}{ccc}
C_{2} q_{x} & D q_{z} & -D q_{y} \\
D q_{z} & -C_{2} q_{x} & 0 \\
-D q_{y} & 0 & -C_{2} q_{x}
\end{array}\right),
$$

where the model parameters $C_{1(2)}, D$ are real and the first term in $\mathcal{H}_{T}$ denotes the tilt effect. Along $q_{x}$ axis $(\Gamma-$ 


\begin{tabular}{|c|c|c|c|c|c|}
\hline$C_{4 v}$ & $E$ & $2 C_{4}$ & $C_{2}$ & $2 \sigma_{v}\left(\mathcal{M}_{x y}\right)$ & $2 \sigma_{d}$ \\
\hline \hline$B_{1}$ & 1 & -1 & 1 & 1 & -1 \\
\hline$E$ & 2 & 0 & -2 & 0 & 0 \\
\hline
\end{tabular}

TABLE I. Character table for the the two encountered representations of $C_{4 v}$ point group on $\Gamma-X$ path.

$X$ path $)$, the dispersion of model $\mathcal{H}_{T}$ is $\varepsilon=\left(C_{1} \pm C_{2}\right) q_{x}$ indicating a triply degenerate point at $q_{x}=0$. Moreover, by fitting of this model to the DFT band structure [see Fig. 2(b)], we find $C_{1}=6.607 \mathrm{eV} \cdot \AA, C_{2}=3.827$ $\mathrm{eV} \cdot \AA$ and $D=0.114 \mathrm{eV} \cdot \AA$. Hence, one has $\left|C_{1}\right|>\left|C_{2}\right|$ which is the typical feature of type-II nodal point. Figure 2(b) also shows the linear fitting works well at least up to $\sim 1 \mathrm{eV}$ energy range. In Fig. 2(c), we plot the dispersion of TNP along the $q_{y}$ direction in the $q_{z}=0$ plane. In contrast, the dispersion along $q_{y}$ direction does not have energy tilt, due to the presence of mirror symmetry $\left(\mathcal{M}_{x z}\right)$ with respect to $x-z$ plane. The dispersion along $q_{z}$ direction is identical with that along $q_{y}$ direction as guaranteed by the $C_{4 x}$ rotation symmetry with respect to $\Gamma-X$ path. The $C_{4 x}$ rotation symmetry also manifests itself in Hamiltonian (1), e.g. the coefficients of $q_{y}$ and $q_{z}$ in $\mathcal{H}_{T}$ are the same (up to their sign).

\section{THREE-BAND NODAL RING}

From Fig. 2(c), one observes that near TNP ( $T$ point) there exist two doubly degenerate points $(R)$, protected by $\mathcal{M}_{x y}$ symmetry as we will discuss later. Due to the presence of $\mathcal{P} \mathcal{T}$ symmetry in $\mathrm{ZrO}$, point $R$ would not exist in isolation but hint the appearance of $\mathrm{NR}^{33}$. In the following, we discuss the NR containing point $R$.

First, we present the band dispersion along $q_{y}$ direction for a constant $q_{x}$ value setting below $\left(q_{x}<0\right)$ and above $\left(q_{x}>0\right)$ the TNP [Fig. 2(d) and 2(e)]. In these two cases, there also exist band crossing points (also labeled as $R$ ), indicating the NR may lie in $k_{z}=0$ plane. Indeed, a careful scan of band dispersion in $k_{z}=0$ plane shows there exists a NR centered at $\Gamma$ point with fourleaf clover-like shape, as shown in Fig. 3(a). Particularly, from Fig. 2(c), one finds the formation of the NR here requires three bands. Hence we term it as TNR. TNR has never been studied in previous works.

Then, we preform a symmetry analysis on the formation of TNR. For simplification, we divide the three relevant bands as $D$ bands and $S$ band, as indicated in Fig. 2(d) and 2(e). $D$ bands are the two bands which would stick together on $\Gamma$ - $X$ line [containing the $q_{y}=0$ point in Fig. 2(c)-2(e)] and $S$ band is the remaining one. With symmetry analysis, one knows: (i), $D$ bands, which are doubly degenerate at $q_{y}=0$ point [Fig. 2(d) and $2(\mathrm{e})]$, would split into two nondegenerate bands at generic momentum points in $q_{z}=0$ plane, as such points do not have $C_{4 v}$ symmetry. (ii), $D$ and $S$ bands are in $q_{z}=0$ mirror plane (containing $\Gamma-X$ line) and hence have explicit eigenvalues of mirror symmetry $\mathcal{M}_{x y}$, given as $n_{M}= \pm 1$. Also, we know the IRs of $S$ and $D$ bands on $\Gamma$ - $X$ line are $B_{1}$ and $E$ [see Fig. 2(a)], and the character for $\mathcal{M}_{x y}$ symmetry of $B_{1}$ and $E$ IRs are 1 and 0 (see Tab. I). Thus, the $\mathcal{M}_{x y}$ eigenvalues of $S$ band and $D$ bands are $n_{M}=1$ and $n_{M}= \pm 1$, respectively. As a result, band crossing can happen between the three bands with different $n_{M}$ and the degeneracy of the crossing points is two-fold, not three-fold as that on $\Gamma-X$ line. By analyzing the mirror eigenvalue of $S$ and $D$ bands in detail, it is found the upper (lower) branch of $D$ bands has $n_{M}=1$ $\left(n_{M}=-1\right)$ while the $S$ band has $n_{M}=1$ [see Fig. 2(d) and 2(e)], consistent with the above discussion.

For the case of $q_{x}<0$ (below TNP), $S$ band is higher than $D$ bands at $q_{y}=0$ point [Fig. 2(d)]. Since $S$ band and $D$ upper band share same mirror eigenvalue $\left(n_{M}=\right.$ 1 ), they would feature anticrossing. Meanwhile, the $D$ bands form a band crossing between themselves as they have opposite $n_{M}$. In sharp contrast, for $q_{x}>0$ (above TNP) , $S$ band is lower than $D$ bands at $q_{y}=0$ point [Fig. 2(e)], then $S$ band can linearly cross with the lower band of $D$. Consequently, the nodal points are formed by the $D$ bands when $q_{x}<0$ and by the $S$ band and the lower band of $D$ when $q_{x}>0$, indicating that the band order inverse between $S$ and $D$ bands at $q_{x}=0$ point ( $\Gamma$ $X$ line) is crucial for the formation of TNR. Observe that this band inversion is inevitable due to the appearance of TNP (see Fig. 2a). Thus, for TNP with dispersion being similar with that in Fig. 2(c), one can expect TNR may coexist with TNP. Indeed, TNP with such dispersion can be found in many identified TNP materials ${ }^{22,44}$ and lots of experimentally synthesized $\mathrm{ZrO}$ family materials (see Figure S1 in Supporting Information).

Since the evolution of two-band and four-band NR under strain are very different ${ }^{45}$, TNR proposed here under strain may also show unique behavior. Figure 3(a-d) show the shape of TNR for four representative hydrostatic strains of $0 . \%, 1.18 \%, 1.2 \%$ and $2 \%$. Figure $3(\mathrm{e}-\mathrm{h})$ give the band dispersion of $\Gamma-P_{1(2)}$ line under the corresponding strains. In Fig. 3(e-h), the relevant three bands are highlighted by red or blue, according to their $\mathcal{M}_{x y}$ mirror eigenvalues $\left(n_{M}= \pm 1\right)$. Here, we only focus on the band crossings between the three bands, e.g. cyan dots in Fig. 3(e-h). Under positive strain, the order of bands at $\Gamma$ point would inverse [see Fig. 3(e) and 3(f)]. This band inversion gives rise to a new nodal ring [see Fig. 3(b)], which can be clearly find from Fig. 3(e-f) as the number of the crossing point on $\Gamma-P_{1(2)}$ line has changed from one [Fig. 3(e)] to two [Fig. 3(f)]. Increasing strain to a critical value $(1.2 \%)$, the new nodal ring would grow in size and finally touch with the original one as shown in Fig. 3(c). Though the two nodal ring are touched together here, they are not nodal chain ${ }^{51-53}$ as the two nodal rings are in the same plane and the touching is accident. Remarkably, beyond the critical strain, the two nodal rings would merge together to produce four droplet-shaped nodal rings [see Fig. 3(d)]. Keep increasing strain, the droplet-shaped nodal rings would 

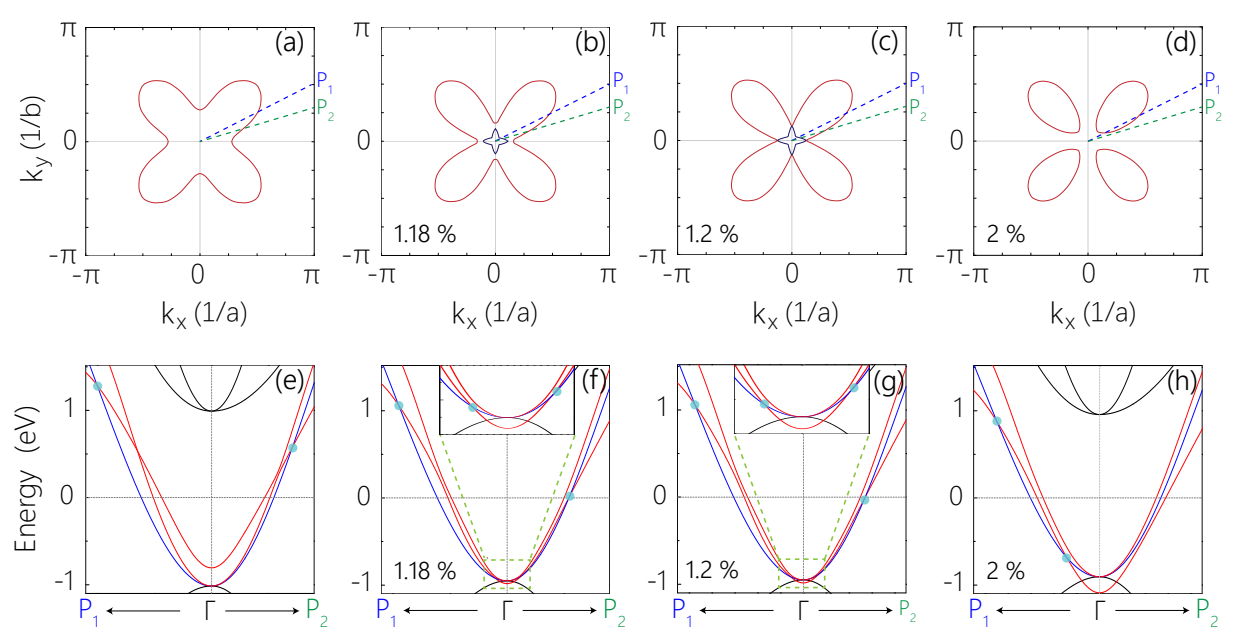

FIG. 3. The shape of TNR of ZrO under the hydrostatic strain of (a) $0 \%$, (b) $1.18 \%$, (c) $1.2 \%$ and (d) $2 \%$. (e-h) Electronic band structures of $\mathrm{ZrO}$ along two paths denoted by blue $\left(\Gamma-P_{1}\right)$ and green $\left(\Gamma-P_{2}\right)$ dashed lines in $(\mathrm{a}-\mathrm{d})$ for different strain. In (e-h), the red lines and blue line are the three bands forming TNR with mirror eigenvalues $n_{M}=1$ and $n_{M}=-1$, respectively. The band crossing points are labeled by the cyan dots.

fade away (not shown). This unique evolution of TNR in $\mathrm{ZrO}$ results from the strong entanglement of the three bands, which can not be found in two-band NR.

\section{TYPE-II DIRAC POINTS}

At last, we discuss the band dispersion of $\mathrm{ZrO}$ with SOC, which is plotted in Fig. 4. Due to the presence of $\mathcal{P}$ and $\mathcal{T}$ symmetry, each band in Fig. 4 is at least doubly degenerate. On $\Gamma-X$ path, the type-II TNP transforms into a type-II Dirac point formed by the bands with $\Gamma_{6}$ and $\Gamma_{7}$ IRs of $C_{4 v}$ double group [Fig. 4(a)]. Also, the dispersion of Dirac node along $q_{y}$ direction in $q_{z}=0$ plane is shown in Fig. 4(b). Due to the $C_{4 x}$ rotation symmetry, the dispersion along $q_{z}$ direction is same with that along $q_{y}$ direction. Using the $\Gamma_{6}$ and $\Gamma_{7}$ states at Dirac point as basis, the low-energy $k \cdot p$ Hamiltonian around Dirac point (up to linear order measured from Dirac point) can be established as

$$
\mathcal{H}_{D}=\left(\begin{array}{cc}
h_{+} & 0 \\
0 & h_{-}
\end{array}\right)
$$

where each entry is a $2 \times 2$ matrix with

$$
h_{ \pm}=w q_{x}+v_{1}\left(q_{z} \sigma_{x} \pm q_{y} \sigma_{y}\right)+v_{2} q_{x} \sigma_{z} .
$$

Here, $\boldsymbol{\sigma}$ are Pauli matrix. The expression of $h_{ \pm}$denotes a tilted Weyl Hamiltonian with \pm 1 chirality, directly showing this band crossing is a Dirac node. Coefficients $v_{1(2)}$ and $w$ represent Fermi velocity and energy tilt, respectively. By fitting Hamiltonian $\mathcal{H}_{D}$ to DFT results [see Fig. $4(\mathrm{c})]$, we find $w=6.607 \mathrm{eV} \cdot \AA, v_{1}=0.343 \mathrm{eV} \cdot \AA$ and $v_{2}=3.925 \mathrm{eV} \cdot \AA$, and have $|w|>\left|v_{2}\right|$ which is consistent with the type-II dispersion. Meanwhile, the TNR and
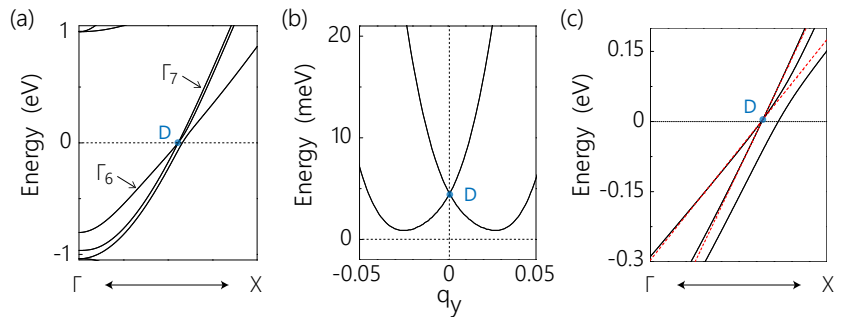

FIG. 4. (a) Band structure on the $\Gamma-X$ line with SOC included. The irreducible representation of bands forming Dirac point are indicated. (b) The band dispersion of Dirac point along the $q_{y}$-direction. (c) Enlargement of electronic band structure around Dirac point by first-principles calculations (solid lines) and $k \cdot p$ model fitting (red dashed lines). The Dirac point is labeled as D.

the band crossing at $\mathrm{W}$ point are gapped (see Figure S2 in Supporting Information).

Compared with previously identified type-II Dirac materials, the type-II Dirac point in $\mathrm{ZrO}$ has several advantages. (i): The Dirac points here almost locate at Fermi energy $(<5 \mathrm{meV})$, facilitating the experimental observation. (ii): The linear energy range of Dirac point along tilt direction is large [see Fig. 4(c)], especially for the valence band $(\sim 0.3 \mathrm{eV})$, offering a good platform for studying the intriguing phenomena associated with type-II dispersion by transport. (iii): All the three pairs of type-II Dirac points are in the same Fermi energy as they are related to each other by symmetries, facilitating the experimental identification of Dirac nodes. 


\section{DISCUSSION AND CONCLUSION}

From the character table of $C_{3 v}, C_{4 v}$ and $C_{6 v}$ point group (without SOC), one knows there exist both oneand two-dimensional IRs and all the doubly degenerate bands exhibit opposite mirror eigenvalues. Thus, according to the above discussion in $\mathrm{ZrO}$, one can expect that TNP and TNR may widely coexist in the materials with these symmetries.

When SOC is included, the appearance of TNP and TNR requires the absence of $\mathcal{P}$ symmetry (assuming $\mathcal{T}$ symmetry maintains). In the character table of $C_{4 v}$ and $C_{6 v}$ double group, only two-dimensional IRs exist. Thus, TNP can not be realized in the rotation axe with $C_{4 v}$ and $C_{6 v}$ symmetries. In contrast, for rotation axe exhibiting $C_{3 v}$ symmetry there still coexist one- and twodimensional IRs and the doubly degenerate band features opposite mirror eigenvalues. Thus, in a spinful system with $C_{3 v}$ symmetry, the TNP may be realized in the rotation axe and the TNR may be found in the mirror plane, provided $\mathcal{P}$ is broken.

Intriguing magneto-transport phenomena have been studied for a variety of nontrivial band crossings and there exists important distinctions between them. First we compare the magneto-transport in type-I and typeII TNP. Due to the energy tilt, the Landau level (LL) spectrum of type-II TNP semimetal is sensitive to the angle between the magnetic field and the energy tilt direction. Particularly, when the angle beyond a critical value, the LL spectrum collapses ${ }^{24}$. Such collapse is a unique feature of type-II band crossing and cannot be found in type-I TNP semimetal. Then comparing type-II TNP and type-II Weyl (Dirac) semimetal, we note that the type-II Weyl (Dirac) point only connects one (two identical) electron pocket(s) and one (two identical) hole pocket(s) ${ }^{12,13,25}$, whereas the type-II TNP would connect three pockets: either two electron pockets and one hole pocket or one electron pocket and two hole pockets, as a TNP is formed by three bands. For example, the TNP shown in Fig. 2(a) connects two electron pockets and one hole pocket. Because the magnetoresponse near type-II
Weyl (Dirac) point and TNP are dominated by the magnetic tunneling between the electron and hole pockets ${ }^{25}$, one can expect that the magnetic quantum oscillations of type-II TNP and type-II Weyl (Dirac) semimetal near nodal point would be very different.

Experimentally, the band features predicted here can be detected by the ARPES technique and transport measurements. Due to the strong metallization of $\mathrm{ZrO}$, the topological surface states are deeply buried in the bulk bands and hence are hard to be detected (see Figure S3 in Supporting Information). However, the bulk type-II Dirac points should be directly observed by ARPES. In addition, since the type-II Dirac node locates around Fermi energy, it should be identified by magneto-transport experiment, as its magnetoresponse is distinguished from type-I semimetal by LL collapses ${ }^{24}$, from type-II Weyl semimetal by anomalous chiral $\mathrm{LLs}^{21}$ and from type-II TNP semimeatal by magnetic Klein tunneling $^{25}$ as discussed above.

In conclusion, we have reported that $\mathrm{ZrO}$ is a novel topological metals. When SOC is ignored, three pairs of type-II TNPs and TNRs coexist in ZrO. The TNR is formed by three bands and its evolution against symmetry-preserving strain distinct from that in twoband NR. Under SOC, each type-II TNP transforms into a Dirac point. Particularly, all the Dirac points reside at same energy and exhibit large linear energy range along their tilt directions, offering facility for detecting the interesting properties induced by type-II dispersion.

\section{ACKNOWLEDGMENTS}

The work is supported by the National Key R\&D Program of China (Grant No. 2016YFA0300600), the MOST Project of China (Grants No. 2014CB920903) and the NSF of China (Grants Nos. 11734003, 11574029).The National Key R\&D program under Grant No. 2016YFA0300904, the National Science Foundation of China under Grant No. 61325021, the Key Research Program of Frontier Sciences, CAS under Grant No. QYZDB-SSW-SLH004, and the Strategic Priority Research Program (B), CAS under Grant No. XDPB0602. *zhiming_yu@sutd.edu.sg

† gyzhang@aphy.iphy.ac.cn

¥ ygyao@bit.edu.cn

1 X.-L. Qi and S.-C. Zhang, Rev. Mod. Phys. 83, 1057 (2011).

2 M. Z. Hasan and C. L. Kane, Rev. Mod. Phys. 82, 3045 (2010).

3 X. Wan, A. M. Turner, A. Vishwanath, and S. Y. Savrasov, Phys. Rev. B 83, 205101 (2011).

${ }^{4}$ G. Xu, H. Weng, Z. Wang, X. Dai, and Z. Fang, Phys. Rev. Lett. 107, 186806 (2011).

${ }^{5}$ H. Weng, C. Fang, Z. Fang, B. A. Bernevig, and X. Dai,
Phys. Rev. X 5, 011029 (2015).

${ }^{6}$ Z. Wang, Y. Sun, X.-Q. Chen, C. Franchini, G. Xu, H. Weng, X. Dai, and Z. Fang, Phys. Rev. B 85, 195320 (2012).

7 Z. Wang, H. Weng, Q. Wu, X. Dai, and Z. Fang, Phys. Rev. B 88, 125427 (2013).

8 X.-L. Sheng, Z. Wang, R. Yu, H. Weng, Z. Fang, and X. Dai, Phys. Rev. B 90, 245308 (2014).

9 W. Wang, L. Deng, N. Jiao, P. Zhou, and L. Sun, physica status solidi (RRL)-Rapid Research Letters 11, 1700271 (2017).

10 S. A. Yang, Spin 06, 1640003 (2016). 
11 S. Guan, Z.-M. Yu, Y. Liu, G.-B. Liu, L. Dong, Y. Lu, Y. Yao, and S. A. Yang, npj Quantum Materials 2, 23 (2017).

12 A. A. Soluyanov, D. Gresch, Z. Wang, Q. Wu, M. Troyer, X. Dai, and B. A. Bernevig, Nature 527, 495 (2015).

13 K. Deng, G. Wan, P. Deng, K. Zhang, S. Ding, E. Wang, M. Yan, H. Huang, H. Zhang, Z. Xu, and et al., Nat Phys 12, 1105 (2016).

14 G. Chang, S.-Y. Xu, S.-M. Huang, D. S. Sanchez, C.H. Hsu, G. Bian, Z.-M. Yu, I. Belopolski, N. Alidoust, H. Zheng, and et al., Sci. Rep. 7, 1688 (2017).

15 P.-J. Guo, H.-C. Yang, K. Liu, and Z.-Y. Lu, Phys. Rev. B 95, 155112 (2017).

16 H. Huang, S. Zhou, and W. Duan, Phys. Rev. B 94, 121117 (2016).

17 B. Bradlyn, J. Cano, Z. Wang, M. G. Vergniory, C. Felser, R. J. Cava, and B. A. Bernevig, Science 353, 5037 (2016).

18 H. Weng, C. Fang, Z. Fang, and X. Dai, Phys. Rev. B 93, 241202 (2016).

19 H. Weng, C. Fang, Z. Fang, and X. Dai, Phys. Rev. B 94, 165201 (2016).

20 J.-P. Sun, D. Zhang, and K. Chang, Phys. Rev. B 96, 045121 (2017).

21 T.-R. Chang, S.-Y. Xu, D. S. Sanchez, W.-F. Tsai, S.-M. Huang, G. Chang, C.-H. Hsu, G. Bian, I. Belopolski, Z.-M. Yu, S. A. Yang, T. Neupert, H.-T. Jeng, H. Lin, and M. Z. Hasan, Phys. Rev. Lett. 119, 026404 (2017).

22 Z. Zhu, G. W. Winkler, Q. Wu, J. Li, and A. A. Soluyanov, Phys. Rev. X 6, 031003 (2016).

23 C. Chen, S.-S. Wang, L. Liu, Z.-M. Yu, X.-L. Sheng, Z. Chen, and S. A. Yang, Phys. Rev. Materials 1, 044201 (2017).

24 Z.-M. Yu, Y. Yao, and S. A. Yang, Phys. Rev. Lett. 117 (2016).

25 T. E. O'Brien, M. Diez, and C. W. Beenakker, Phys Rev Lett 116, 236401 (2016).

26 A. Tamai, Q. S. Wu, I. Cucchi, F. Y. Bruno, S. Riccò, T. K. Kim, M. Hoesch, C. Barreteau, E. Giannini, C. Besnard, and et al., Phys. Rev. X 6, 031021 (2016).

27 H.-J. Noh, J. Jeong, E.-J. Cho, K. Kim, B. I. Min, and B.-G. Park, Phys. Rev. Lett. 119, 016401 (2017).

${ }^{28}$ M. Yan, H. Huang, K. Zhang, E. Wang, W. Yao, K. Deng, G. Wan, H. Zhang, M. Arita, H. Yang, Z. Sun, H. Yao, Y. Wu, S. Fan, W. Duan, and S. Zhou, Nat. Comm. 8, 257 (2017).

${ }^{29}$ K. Zhang, M. Yan, H. Zhang, H. Huang, M. Arita, Z. Sun, W. Duan, Y. Wu, and S. Zhou, Phys. Rev. B 96, 125102 (2017).

${ }^{30}$ F. Fei, X. Bo, R. Wang, B. Wu, J. Jiang, D. Fu, M. Gao, H. Zheng, Y. Chen, X. Wang, and et al., Phys. Rev. B 96, 041201 (2017).
31 B. Q. Lv, Z. L. Feng, Q. N. Xu, X. Gao, J. Z. Ma, L. Y. Kong, P. Richard, Y. B. Huang, V. N. Strocov, C. Fang, H. M. Weng, Y. G. Shi, T. Qian, and H. Ding, Nature 546, 627 (2017).

32 J. Ruan, S.-K. Jian, D. Zhang, H. Yao, H. Zhang, S.-C. Zhang, and D. Xing, Phys. Rev. Lett. 116, 226801 (2016).

33 H. Weng, Y. Liang, Q. Xu, R. Yu, Z. Fang, X. Dai, and Y. Kawazoe, Phys. Rev. B 92, 045108 (2015).

34 R. Yu, H. Weng, Z. Fang, X. Dai, and X. Hu, Phys. Rev. Lett. 115, 036807 (2015).

35 Y.-H. Chan, C.-K. Chiu, M. Y. Chou, and A. P. Schnyder, Phys. Rev. B 93, 205132 (2016).

36 S. Li, Z.-M. Yu, Y. Liu, S. Guan, S.-S. Wang, X. Zhang, Y. Yao, and S. A. Yang, Phys. Rev. B 96, 081106 (2017).

37 R. Li, H. Ma, X. Cheng, S. Wang, D. Li, Z. Zhang, Y. Li, and X.-Q. Chen, Phys. Rev. Lett. 117, 096401 (2016).

38 X.-L. Sheng, Z.-M. Yu, R. Yu, H. Weng, and S. A. Yang, J. Phys. Chem. Lett. 8, 3506 (2017).

39 G. Bian, T.-R. Chang, R. Sankar, S.-Y. Xu, H. Zheng, T. Neupert, C.-K. Chiu, S.-M. Huang, G. Chang, I. Belopolski, and et al., Nat. Comm. 7, 10556 (2016).

${ }^{40}$ L. M. Schoop, M. N. Ali, C. Straer, A. Topp, A. Varykhalov, D. Marchenko, V. Duppel, S. S. P. Parkin, B. V. Lotsch, and C. R. Ast, Nat. Comm. 7, 11696 (2016).

41 C. Zhong, Y. Chen, Z.-M. Yu, Y. Xie, H. Wang, S. A. Yang, and S. Zhang, Nat. Comm. 8, 15641 (2017).

42 X. Zhang, L. Jin, X. Dai, and G. Liu, J. Phys. Chem. Lett. 8, 4814 (2017).

43 G. Chang, S.-Y. Xu, X. Zhou, S.-M. Huang, B. Singh, B. Wang, I. Belopolski, J. Yin, S. Zhang, A. Bansil, H. Lin, and M. Z. Hasan, Phys. Rev. Lett. 119, 156401 (2017).

44 X. Zhang, Z.-M. Yu, X.-L. Sheng, H. Y. Yang, and S. A. Yang, Phys. Rev. B 95, 235116 (2017).

45 C. Fang, Y. Chen, H.-Y. Kee, and L. Fu, Phys. Rev. B 92, $081201(2015)$.

46 G. Kresse and J. Furthmller, Computational Materials Science 6, 15 (1996).

47 P. E. Blöchl, Phys. Rev. B 50, 17953 (1994).

48 J. P. Perdew, J. A. Chevary, S. H. Vosko, K. A. Jackson, M. R. Pederson, D. J. Singh, and C. Fiolhais, Phys. Rev. B 46, 6671 (1992).

49 N. Schonberg, Acta Chemica Scandinavica 8, 627 (1954).

50 P. Moseley and B. Hudson, Journal of Nuclear Materials 99, 340 (1981).

51 T. Bzdusek, Q. Wu, A. Regg, M. Sigrist, and A. A. Soluyanov, Nature 538, 75 (2016).

52 Wang, S.-S.; Liu, Y.; Yu, Z.-M.; Sheng, X.-L.; Yang, S. A. Hourglass Dirac Chain Metal in Rhenium Dioxide. 2017, arXiv:1705.01424.

53 R. Yu, Q. Wu, Z. Fang, and H. Weng, Phys. Rev. Lett. 119, 036401 (2017). 